\title{
Immune Checkpoint Inhibitors in MSI-H/dMMR Colorectal Cancer: A Mini-Review
}

\author{
Ali Sanjari Moghaddam ${ }^{1,2}$, Roham Salman Roghani ${ }^{1,2}$ and David S Hsu ${ }^{1,2 *}$ \\ ${ }^{1}$ Department of Medicine, Division of Medical Oncology, Duke University Medical Center, Durham, North Carolina, USA \\ ${ }^{2}$ Duke Cancer Institute, Duke University, Durham, North Carolina, USA
}

*Corresponding author: David S Hsu, 3008 Snyderman Building, 905 S. LaSalle St., Duke University, Medical Center, Durham, NC 27710, USA.

Received Date: July 13, 2020

Published Date: July 27, 2020

\begin{abstract}
Microsatellite instability-high/mismatch repair deficient (MSI-H/dMMR) colorectal cancer accounts for $15 \%$ of all colorectal cancer cases and $5 \%$ of metastatic colorectal cancer. Although cancer immunotherapy has been effective in various cancer, its activity has been limited in colorectal cancer. However, cancer immunotherapy has been shown to be effective in MSI-H/dMMR colorectal cancer. Specifically, among immunotherapeutic modalities, immune checkpoint inhibitors have shown the most promising results in MSI-H/dMMR colorectal cancer and to date, the Food and Drug Administration (FDA) has approved three checkpoint inhibitors for the treatment of metastatic MSI-H/dMMR colorectal cancer. With the recent advances, checkpoints inhibitors can now be considered as the first line of therapy in advanced dMMR/MSI-H colorectal cancer, and in this review, we will examine the development and use of immune checkpoint inhibitors in the treatment of metastatic colorectal cancer.
\end{abstract}

Keywords: Colorectal cancer; Immunotherapy; Immune Checkpoint Inhibitor; Microsatellite instability; Mismatch repair deficiency

Abbreviations: MSI-H/dMMR: Microsatellite instability-high/mismatch repair; FDA: Food and Drug Administration; ORR: Objective response rate; PFS: Progression-free survival; OS: Overall survival

\section{Introduction}

Colorectal cancer with an estimated 147,950 new cases in 2020 is the fourth most common cancer in the United States and with about estimated 53,000 death in 2020 stands as the second most lethal cancer in the United States. Due to colorectal cancer screening, colorectal cancer-related mortality has been decreasing, but at the time of diagnosis, $22 \%$ of patients present with advanced metastatic disease with a 5 -year survival of $<10 \%$ [1]. Colorectal cancer can be classified as either microsatellite instability-high/ mismatch repair deficient (MSI-H/dMMR) or microsatellite stable tumor (MSS). MSI-H/dMMR tumors account for approximately $15 \%$ of all colorectal cancer cases and $5 \%$ of metastatic cases [2]. The MSI-H/dMMR phenotype results from a deficiency in the mismatch repair pathway [3]. Approximately eighty percent of MSI-H/dMMR colorectal cancer are sporadic and result from either somatic mutation or most commonly hypermethylation of promotor region of DNA mismatch repair gene such as MLH1. The remaining twenty percent results from a germline mutation in DNA mismatch repair genes such as MLH1, MSH2, MSH6, and PMS2 and constitutes the Lynch syndrome [4]. Recently, MSI-H/dMMR has been shown to be a predictor of response to immunotherapy [5]. It has been proposed that defective expression of mismatch repair gene and high microsatellite instability lead to high mutational burden and neoantigen presentation, and subsequent infiltration of tumor with immune cells [6]. The high level of neoantigen presentation and strong antitumor immune response make MSI-H/ dMMR colorectal cancer a good candidate for immunotherapy, particularly immune checkpoint inhibitors. Currently, there are 
three immune checkpoint inhibitors that have been FDA approved for the treatment of metastatic colorectal cancer, ipilimumab, pembrolizumab, and nivolumab. Ipilimumab, which targets CTLA4, is approved for the treatment of MSI-H/dMMR metastatic colorectal cancer that has progressed following treatment with a fluoropyrimidine, oxaliplatin, and irinotecan, in combination with nivolumab. Pembrolizumab, which targets PD-1, is approved for the treatment of MSI-H/dMMR metastatic colorectal cancer that has progressed following treatment with a fluoropyrimidine, oxaliplatin, and irinotecan or who have no satisfactory alternative treatment options. Nivolumab, which targets PD-1, is approved for the treatment of MSI-H/dMMR metastatic colorectal cancer that has progressed following treatment with a fluoropyrimidine, oxaliplatin, and irinotecan, as a single agent or in combination with ipilimumab.

\section{Pembrolizumab}

Pembrolizumab was the first immune checkpoint inhibitor to receive FDA approval for the treatment of metastatic MSI-H/dMMR colorectal cancer in 2017 [7]. Le and colleagues in a phase II clinical trial assessed the effectiveness of pembrolizumab for the treatment of progressive metastatic carcinoma with or without mismatchrepair deficiency. Overall, the study showed that immune-related objective response rate (ORR) and immune-related progressionfree survival (PFS) rate for MSI/dMMR tumors were $40 \%$ and $78 \%$, and $0 \%$ and $11 \%$ for MSS tumor, respectively. PFS and overall survival (OS) were 2.2 and 5 months for MSS colorectal cancer but have not been reached for MSI colorectal cancer [8]. KEYNOTE-164 (NCT02460198), was a phase II clinical trial of patients with MSI-H/dMMR advanced colorectal cancer with progressive disease after treatment with a fluoropyrimidine, oxaliplatin, and irinotecan. There were two cohorts in the study with one cohort (A) receiving two lines of the standard of care therapy and the other (B) receiving only one line of therapy. This study showed an ORR of 33\% in both cohorts with median PFS and OS of 2.3 and 31.4 months for cohort A and in cohort B, median PFS was 4.1 months but OS has not been reached [9]. KEYNOTE-177 (NCT02563002) was a phase III randomized trial on patients evaluating KEYTRUDA monotherapy versus standard of care chemotherapy for the first-line treatment of patients with MSI-H/dMMR advanced colorectal cancer and was recently presented at the plenary session at the ASCO Annual Meeting (2020). In this study, PFS and OR were found to be 16.5 months and $43.8 \%$ for pembrolizumab, and 8.2 months and $33.1 \%$ for chemotherapy. Results of KEYNOTE-177 demonstrated a significant clinical superiority of immune checkpoint inhibitor, pembrolizumab over chemotherapy for patients with MSI-H/ dMMR colorectal cancer [10].

\section{Nivolumab and Ipilimumab}

Nivolumab was first shown to have activity in MSI-H/dMMR colorectal cancer in a phase I trial of refractory solid tumors where one patient with MSI-H/dMMR colorectal cancer had a complete response of 21 months [11]. This led to CheckMate142, a multicenter phase 2 trial (NCT02060188), where Overman and colleagues studied the effect of nivolumab on dMMR/MSI-H recurrent or metastatic colorectal cancer on patients with no response or intolerance to fluoropyrimidine and oxaliplatin or irinotecan. After 12 months of follow up, 31\% of patients showed an objective response and $69 \%$ of patients had stable disease for at least 12 weeks [12]. The results of this study led to FDA approval of nivolumab for the treatment of dMMR/MSI-H advanced colorectal cancer in 2017. Overman and colleagues then extended the CheckMate142 study to assess the efficacy of the combination of two checkpoint inhibitors in advanced dMMR/MSI-H colorectal cancer. Following initial treatment with four doses of nivolumab plus ipilimumab, patients were continued to be given nivolumab every 2 weeks. The results showed an ORR of 55\% and more than 12 weeks of stable disease in $80 \%$ of patients after 13.4 months of follow up. Twelve-month PFS and OS were $71 \%$ and $85 \%$, respectively. It was concluded that a combination of nivolumab and ipilimumab potentially improves the patient's outcome compared to nivolumab as a monotherapy [13]. As a result of this study, the FDA approved nivolumab/Ipilimumab for the treatment of metastatic dMMR/MSI-H colorectal cancer in 2018.

\section{Conclusion}

Currently, immune checkpoint inhibitors have been demonstrated to have clinical benefit in the treatment of dMMR/ MSI-H colorectal cancer. Furthermore, the era of immunotherapy is moving forward in colorectal cancer and many clinical trials investigating the role of new immune checkpoint inhibitors such as atezolizumab (NCT02997228) and Durvalumab (NCT02227667) in dMMR/MSI-H colorectal cancer is underway. Currently, three immune checkpoint inhibitors including pembrolizumab, nivolumab, and ipilimumab are approved for the treatment of advanced dMMR/MSI-H colorectal cancer for the patients who have progressed on at least one line of therapy. However, with newly published results of KEYNOTE-177, checkpoints inhibitors can now also be potentially considered as the first line of therapy in advanced dMMR/MSI-H colorectal cancer.

\section{Acknowledgement}

None.

\section{Conflict of Interest}

No conflicts of interest.

\section{References}

1. Siegel RL, Miller KD, Jemal A (2020) Cancer statistics. CA Cancer J Clin 70: 7-30.

2. Colle R, Cohen R, Cochereau D, Duval A, Lascols O, et al. (2017) Immunotherapy and patients treated for cancer with microsatellite instability. Bulletin du cancer 104(1): 42-51. 
3. Eso Y, Shimizu T, Takeda H, Takai A, Marusawa H (2020) Microsatellite instability and immune checkpoint inhibitors: toward precision medicine against gastrointestinal and hepatobiliary cancers. Journal of gastroenterology 55(1): 15-26.

4. Gatalica Z, Vranic S, Xiu J, Swensen J, Reddy S (2016) High microsatellite instability (MSI-H) colorectal carcinoma: a brief review of predictive biomarkers in the era of personalized medicine. Fam Cancer 15(3): 405412.

5. Hewish M, Lord CJ, Martin SA, Cunningham D, Ashworth A (2010) Mismatch repair deficient colorectal cancer in the era of personalized treatment. Nat Rev Clin Oncol 7(4): 197-208.

6. Zhao P, Li L, Jiang X, Li Q (2019) Mismatch repair deficiency/microsatellite instability-high as a predictor for anti-PD-1/PD-L1 immunotherapy efficacy. J Hematol Oncol 12(1): 54.

7. Marcus L, Lemery SJ, Keegan P, Pazdur R (2019) FDA Approval Summary: Pembrolizumab for the Treatment of Microsatellite Instability-High Solid Tumors. Clin Cancer Res 25(13): 3753-3758.

8. Le DT, Uram JN, Wang H, Bartlett BR, Kemberling H, et al. (2015) PD-1 Blockade in Tumors with Mismatch-Repair Deficiency. N Engl J Med 372(26): 2509-2520.

9. Shiina S, Shiraishi T (1988) Current significance of physiological function tests--with special reference to cor pulmonale. Rinsho Byori 36(5): 559-560.

10. Andre T, Kai-Keen S, Tae Won K, Benny Vittrup J, Lars Henrik J, et al. (2020) Pembrolizumab versus chemotherapy for microsatellite instability-high/mismatch repair deficient metastatic colorectal cancer: The phase 3 KEYNOTE-177 Study. Journal of Clinical Oncology 38: LBA4LBA4.

11. Brahmer JR, Drake CG, Wollner I, Powderly JD, Picus J, et al. (2010) Phase I study of single-agent anti-programmed death-1 (MDX-1106) in refractory solid tumors: safety, clinical activity, pharmacodynamics, and immunologic correlates. Journal of clinical oncology 28(19): 31673175 .

12. Overman MJ, Dermott RMc, Leach JL, Lonardi S, Lenz HJ, et al. (2017) Nivolumab in patients with metastatic DNA mismatch repair-deficient or microsatellite instability-high colorectal cancer (CheckMate 142): an open-label, multicentre, phase 2 study. Lancet Oncol 18(9): 1182-1191.

13. Overman MJ, Lonardi S, Wong KYM, LenzHJ, Gelsomino F, et al. (2018) Durable Clinical Benefit With Nivolumab Plus Ipilimumab in DNA Mismatch Repair-Deficient/Microsatellite Instability-High Metastatic Colorectal Cancer. J Clin Oncol 36(8): 773-779. 